Revue

Revue de l'histoire des religions

de Ihistoire des religions

2| 2008

La mort et l'émotion. Attitudes antiques

\title{
La mort et l'émotion. Attitudes antiques
}

Avant-propos

Philippe Borgeaud

\section{CpenEdition}

Journals

Édition électronique

URL : http://journals.openedition.org/rhr/6143

DOI : $10.4000 /$ rhr.6143

ISSN : 2105-2573

Éditeur

Armand Colin

Édition imprimée

Date de publication : 1 avril 2008

Pagination : 155-162

ISSN : 0035-1423

\section{Référence électronique}

Philippe Borgeaud, «La mort et l'émotion. Attitudes antiques », Revue de l'histoire des religions [En

ligne], 2 | 2008, mis en ligne le 01 avril 2011, consulté le 21 septembre 2020. URL : http://

journals.openedition.org/rhr/6143; DOI : https://doi.org/10.4000/rhr.6143 


\title{
La mort et l'émotion Attitudes antiques
}

\author{
Avant-propos
}

La thématique des émotions fait partie du champ traditionnel des études religieuses. Depuis William Robertson Smith (The Religion of the Semites, 1889) et Émile Durkheim (Les formes élémentaires de la vie religieuse. Le système totémique en Australie, 1912), les recherches sur le rite ont relevé l'importance des affects dans la constitution (religieuse) du lien social. La fête et son effervescence sont devenues un motif essentiel dans l'approche du sacré effectuée au sein de l'école sociologique française. Parallèlement, William James (dans une perspective psychologique, The Varieties of Religious Experience: a Study in Human Nature, 1902) et Rudolf Otto (dans une posture philosophique et théologique inspirée de Schleiermacher, Das Heilige, 1912) ont contribué eux aussi à l'étude du sacré sous l'angle des émotions. La fondation émotionnelle des comportements rituels n'a pas cessé, non plus, de retenir l'attention des anthropologues, comme en témoignent notamment les travaux de Catherine Lutz et Lila Abu-Lughod ${ }^{1}$. L'histoire des religions est tributaire de l'ensemble de ces travaux.

1. Cf. Lila Abu-Lughod, Veiled Sentiments. Honor and Poetry in a Bedouin Society, Berkeley, University of California Press, 1986 ; Catherine Lutz and Geoffrey M. White, «The Anthropology of Emotions », Annual Review of Anthropology, 15 (1986), p. 405-436 ; Catherine Lutz, Unnatural Emotions. Everyday Sentiments on a Micronesian Atoll and Their Challenge to Western Theory, Chicago, The University of Chicago Press, 1988 ; Catherine Lutz and 
Les comportements et les croyances face à la mort, les coutumes funéraires, constituent à coup sûr un champ d'observation privilégié pour l'étude des émotions religieuses dans leurs rapports aux rites et à l'autorité du sacré. Le déséquilibre que la souffrance et la mort introduisent à l'intérieur d'une collectivité apparaît à l'évidence comme un des facteurs essentiels qui détermine la production de discours et de pratiques religieuses. Dans une perspective anthropologique lointainement inspirée de Spinoza, on dira que le groupe perturbé par le deuil, pour persévérer dans son être, se doit de produire des instruments d'adaptation, voire de survie ${ }^{2}$. Dans une perspective plus historienne on se plaira à observer comment diverses constructions culturelles de la mort et des émotions viennent informer les attitudes observées, ici et là, dans le rapport des individus à leur finitude ${ }^{3}$.

Les études ici réunies abordent leur objet, chacune à sa manière, selon ces deux perspectives. Elles se penchent sur une zone culturelle à la fois très diversifiée et relativement homogène qui s'étend, dans l'Antiquité, de la Mésopotamie au monde « classique », en passant par la vallée du Nil. Toutes abordent un aspect spécifique du deuil dans son lien au sacré, et toutes sont attentives à la coloration particulière, plus ou moins conventionnelle et localement coutumière, que prennent, dans tel ou tel contexte, les émotions manifestées face à la mort, celle d'un proche ou celle (imaginée) de soi-même. Chacune d'elle pose à ce sujet des questions qui font écho aux questions posées par les autres, dans la mesure où elles ont été élaborées dans le cadre d'une réflexion collective sur la ritualisation des émotions. Elles sont en effet le résultat d'un atelier sur «Mythes et rites » organisé par une petite équipe de recherche de l'Université

Lila Abu-Lughod, Language and the Politics of Emotion, Cambridge, Cambridge University Press, 1990 ; John Corrigan (éd.), Religion and Emotion. Approaches and Interpretations, Oxford-New York, Oxford University Press, 2004.

2. Cf. Antonio R. Damasio, Spinoza avait raison. Joie et tristesse, le cerveau des émotions, Odile Jacob (Poches), 2005, p. 278.

3. Cf. Gary L. Ebersole, " Death », in Lindsay Jones (éd.), Encyclopedia of Religion, Second Edition, vol. 4, New York, Macmillan, 2005, p. 2236-2245 (avec bibliographie). 
de Genève dans le cadre du Centre interdisciplinaire en sciences affectives ${ }^{4}$.

D'une étude à l'autre certaines thématiques sont récurrentes, ainsi celle des composantes élémentaires, plus ou moins variables entre Mésopotamie, Égypte, Grèce et Rome, d'un scénario émotionnel complexe. Quatre des six études ici publiées font référence aux travaux d'Ernesto De Martino, notamment pour examiner ce que devient, dans différents champs antiques précisément contextualisés, la succession « stupeur silencieuse », « douleur spontanée », «prise en charge rituelle et collective de la douleur $»$. Cette dramaturgie correspond d'une part à une prise de conscience graduelle de la métamorphose du corps de l'autre en un cadavre à traiter rituellement, et d'autre part à la prise en charge, également graduelle, du deuil individuel par la communauté. On voit se développer, dans chaque culture abordée, des procédures gestuelles très précises, d'abattement, de silence, de prostration, d'agitation, de hurlements, de souillures, de violences (automutilations) et enfin de pleurs collectifs.

Alors même que de telles pratiques peuvent sembler relativement constantes, des questions centrales comme celle des lamentations rituelles et des pleureurs ou pleureuses professionnels (ou non) se posent en des termes différents, d'une culture à l'autre. En Mésopotamie, un nom peut désigner certains spécialistes des pleurs et des lamentations, ce qui n'est le cas ni en Égypte, ni en Grèce et à Rome (où la fonction existe néanmoins). En Égypte les pleureuses répètent les gestes d'Isis et de Nephthys face au cadavre d'Osiris, auquel, d'une certaine manière, elles vont redonner vie. En Grèce et à Rome, le spectacle du deuil répond à d'autres intentions, qui sont plutôt de séparer définitivement les vivants et les morts, ou d'assurer aux vivants une possibilité de continuer à vivre normalement, au-delà du déséquilibre collectif que cause la disparition d'un membre de la communauté.

4. Le CISA, sous l'égide du National Centre of Competence in Research (NCCR) "Affective sciences: Emotions in individual behaviour and social processes ». Project 12: Myths and Rites as Cultural expression of Emotion. 
Deuil et regret, deuil et désir, ce rapport étroit, sentimental, introduit dans la mort une composante sexuelle que l'on ne sera pas surpris de rencontrer aussi bien en Égypte qu'en Mésopotamie, comme le montrent les contributions d'Anne-Caroline Rendu et de Youri Volokhine. On pourrait sur ce point prolonger l'enquête du côté du monde grec, où les rapports étroits de Perséphone et d'Aphrodite, notamment à Locres Épizéphyrienne, ont retenu récemment l'attention de James Redfield ${ }^{5}$.

Anne-Caroline Rendu se réfère à Nicole Loraux pour suggérer que la formalisation sociale, voire la codification littéraire, ne constitue pas un obstacle infranchissable à la perception de ce qui fut, aussi loin de nous dans le temps et la culture que le sont Akkad ou Sumer, l'intime d'une personne. Les trois exemples qu'elle aborde avec autant d'acuité philologique que de sensibilité sont tirés de trois textes majeurs de la littérature mésopotamienne, décrivant tour à tour la douleur de Gilgameš face au cadavre de son ami Enkidu, celle de Geštinnana face à celui de son frère Dumuzi, et enfin celle de la déesse créatrice de l'humanité, Nintu/Mami, face aux corps de ses créatures flottant comme des poissons dans les eaux du déluge.

Youri Volokhine prend appui aussi bien sur les approches classiques de l'école sociologique française (notamment Emile Durkheim, Robert Herz, Maurice Halbwachs et Marcel Mauss), que sur l'anthropologie contemporaine. Son état des lieux met l'accent sur la mise en scène de jeux liturgiques dans son rapport à une idéologie de la mort, en Égypte ancienne. La présentation des rituels liés au deuil de l'Apis introduit au motif du dieu qui meurt, et à celui du trouble et des dangers qui accompagnent la mort du roi. Même les dieux peuvent alors être représentés en deuil, prostrés, la tête sur les genoux. Ce motif de l'affliction divine sous-tend le modèle de toute plainte funèbre, à savoir la quête du disparu, l'être aimé victime d'un crime, puis la déploration d'Osiris par son épouse. La mort, toute mort, apparaît donc assimilée à un meurtre, et fait l'objet d'une lamentation idéalement destinée à réveiller le cadavre. Quelle place,

5. James M. Redfield, The Locrian Maidens. Love and Death in Greek Italy, Princeton University Press, Princeton and London, 2003. Dans d'autres contextes, le regret du mort peut conduire à l'élaboration d'une image, suivie d'un culte. 
dans tout cela, pour des émotions «intimes »? La codification, la dramaturgie, laissent-elles s'exprimer les affects personnels, au-delà de ce que Marcel Mauss appelait l' « expression obligatoire des sentiments »? Rien ne prouve toutefois, selon Y. Volokhine, que ces expressions obligatoires ne sont que de façade.

Claire-Françoise de Roguin nous convie à son tour, comme auparavant Anne-Caroline Rendu, à revisiter un grand modèle littéraire. Son parcours de l'Iliade la conduit à montrer comment Achille fait usage de la pensée, de son noos, dans un processus de réflexion sur l'émotion, inhérent à l'émotion qui traverse tout le poème. Ce processus conduit Achille, à partir de sa colère initiale et d'un second courroux plus vaste encore, mais dirigé sur un objet différent, à maîtriser finalement sa violence et son chagrin, à mettre un frein à l'émotion (sans pour autant la supprimer), au terme d'une réévaluation qui s'élabore entre les funérailles de Patrocle et la visite de Priam venu rechercher le corps d'Hector. Achille et Priam pleurent ensemble. Les pleurs d'Achille sont adressés à sa propre mortalité (comme finalement ceux de Gilgameš, dans l'épopée mésopotamienne).

Sur un plan anthropologique autant que sous l'angle d'une histoire du droit athénien, David Bouvier aborde la question de la législation qui s'efforce de modérer les démonstrations de douleur lors des deuils. Son analyse conduit à réaffirmer l'originalité de la conception platonicienne, en la distinguant de ce que signifie, en général, le désir de contrôler les manifestations (musicales) de la douleur. Les témoignages épigraphiques sur les plaintes funèbres, notamment, invitent à ne pas se fier trop vite à Solon pour imaginer une condamnation radicale des thrènes. Ce qui fait problème, c'est la difficile distinction entre la plainte et l'éloge funèbre, entre le thrène et l'hymne. On rencontre ici un jeu d'oppositions dont David Bouvier, dialoguant avec Nicole Loraux, nous montre qu'il est différent, dans la cité grecque, de ce qu'il peut être à Rome.

Doralice Fabiano nous conduit dans l'Hadès des châtiments, où la peine équivaut à la répétition pénible d'une action sans résultat. Non loin de Sisyphe et des Danaïdes, mais un peu à l'écart, l'énigmatique Oknos apparaît comme un artisan maudit dont l'âne dévore la 
corde à mesure qu'il la tisse. L'enquête minutieuse de Doralice Fabiano montre que les Anciens, en élaborant cette image infernale, furent sensibles à une crainte moins sociale, plus individuelle, plus personnelle liée à la mort : celle de la paralysie, de l'immobilité, de la fixation. Une peur paralysante, causée par une puissance qui lie, menace particulièrement ceux qui ont négligé de se faire initier aux Mystères. Cette négligence des préparatifs à la mort est désignée par le nom même d'Oknos, qui renvoie à l'hésitation, à la procrastination.

Francesca Prescendi montre comment le deuil romain (le luctus) a pu être considéré par les auteurs anciens comme une émotion, un affect perturbateur. L'individu en deuil recherche la cause de sa douleur pour découvrir qu'il est blessé, non pas comme une victime le serait par un adversaire, mais par la condition humaine. La réflexion romaine s'éloigne ici de la conception égyptienne (la mort résultant d'un meurtre) pour rejoindre, partiellement, la leçon de Gilgameš et celle d'Achille. À Rome, on décrit aussi cette stupeur silencieuse (analysée par De Martino) qui précède la lamentation. Celle-ci, comme ailleurs, est hautement ritualisée, thêâtralisée. Toutefois, comme le souligne Francesca Prescendi, cette mise en scène n'est pas simplement une prise en charge collective et un contrôle codifié de la douleur individuelle : elle est à son tour productrice d'affects; le rite suscite lui aussi, au niveau spectaculaire, de l'émotion.

Depuis une vingtaine d'années, de nouveaux développements, impliquant le champ des émotions, introduisent peu à peu au centre de l'horizon transdisciplinaire de l'histoire des religions quelques propositions de la philosophie cognitive, elles-mêmes partiellement tributaires des apports des neurosciences et d'autres disciplines ayant pour objet les affects. Les travaux de Dan Sperber, de Pascal Boyer et de Harvey Whitehouse, dans ce contexte, ont rendu quasi populaires certaines hypothèses sur la diffusion «épidémiologique » des idées ou des représentations (notamment religieuses). L'imagerie cérébrale se trouve invoquée, à son tour et de plus en plus, surtout dans des travaux de vulgarisation, comme un puissant moyen de persuasion pour illustrer ce que l'on pense être, «tout au fond», une émotion religieuse. Il s'agit là d'un vaste chantier, ambitieux, 
visant ni plus ni moins à une théorie universaliste des fondements de l'imaginaire social ${ }^{6}$.

Par rapport aux recherches effectuées dans une telle perspective, une histoire des religions beaucoup moins généraliste, mais résolument comparatiste, et centrée sur la dimension sociale des représentations collectives, garde toute sa raison d'être. Sa démarche consiste à décrire et à explorer de manière empathique les formes diversifiées de son objet, en les envisageant dans l'histoire et dans une perspective anthropologique. Cet objet, en effet, relève du domaine des productions culturelles. Telle qu'elle est pratiquée dans les études qu'on va lire, l'histoire des religions n'a pas pour intention première de dévoiler les ruses de l'esprit (mind en anglais), ni d'examiner les effets d'un environnement ou d'un fondement biologique et physiologique. Elle a encore moins l'ambition d'expliquer les émotions qu'elle rencontre par le fonctionnement du système endocrinien ou neuronal. Les disciplines que cela concerne pourront cependant lui trouver quelque utilité. Avant d'être en mesure de décrire en termes de réaction chimique, ou de «localiser» dans le cerveau une expérience du type «deuil », il peut sembler judicieux de s'être interrogé sur ce qu' on appelle le « deuil », et sur le rapport que ce concept multiforme entretient, en termes de constructions imaginaires, avec les expériences vécues dans tel ou tel contexte culturel. Aucune discipline théorique ne pourra faire l'économie de cette approche «empirique », de cet exercice d'observation, de description et d'analyse, attentif à la diversité des productions discursives (mythologies populaires ou théologies érudites) et généralement symboliques (rites et coutumes).

6. Cf. notamment E. Thomas Lawson and Robert N. McCauley, Rethinking Religion: Connecting Cognition and Culture (1990) ; Harvey Whitehouse, Inside the Cult: Religious Innovation and Transmission in Papua New Guinea, Oxford, Oxford University Press, 1995 ; Dan Sperber, La contagion des idées, Paris, Odile Jacob, 1996 ; Pascal Boyer, Et l'homme créa les dieux. Comment expliquer la religion, Paris, Robert Laffond, 2001 ; E. Thomas Lawson and Robert N. McCauley, Bringing Ritual to Mind: Psychological Foundations of Ritual Forms (2002). Il existe depuis quelque temps une association internationale pour l'étude cognitive des religions (http://www.iacsr.com) ; plusieurs spécialistes de l'Antiquité classique en font partie, comme Roger Beck, Pachis Panayotis et Luther H. Martin. 
En conclusion, nous aimerions dédier cet atelier à la mémoire de Jean Bottéro, qui nous a quittés en décembre 2007, un très grand savant et un ami, qui encouragea autrefois, à Genève, ce type de recherche.

philippe.borgeaud@lettres.unige.ch 